\section{Coffee and gastric cancer: systematic review and meta-analysis}

\author{
Café e câncer gástrico: \\ revisão sistemática e meta-análise
}

\author{
1 Faculdade de Medicina \\ Universidade do Porto, \\ Porto, Portugal. \\ Correspondence \\ H. Barros \\ Serviço de Higiene \\ e Epidemiologia, \\ Faculdade de Medicina, \\ Universidade do Porto. \\ Alameda Prof. Hernâni \\ Monteiro 4200-319, \\ Porto, Portugal. \\ hbarros@med.up.pt
}

\begin{abstract}
We systematically reviewed the literature on the association between coffee consumption and gastric cancer and performed a meta-analysis of the results. Published cohort and case-control studies were identified in PubMed and reference lists. Random effects meta-analysis was used to pool effects from 23 studies, and heterogeneity was explored by stratification and meta-regression. The odds ratio (OR) for the overall association between coffee and gastric cancer (highest vs. lowest category of exposure) was 0.97 (95\% CI: 0.86-1.09), similar for cohort (OR = 1.02; 95\%CI: 0.76 -1.37) and case-control studies (populationbased: $O R=0.90 ; 95 \% C I: 0.70-1.15$; hospitalbased: $O R=0.97 ; 95 \% C I: 0.83-1.13)$. The OR was 1.26 (95\%CI: 1.02-1.57) when considering five studies conducted in the USA, 0.97 (95\%CI: 0.821.14) for the five Japanese studies, 0.98 (95\%CI: 0.81-1.17) for the six studies from Europe, and 0.64 (95\%CI: 0.47-0.86) for the two studies from South America. In this meta-analysis we found no adverse effect of coffee associated with gastric cancer. Knowledge on the level of exposure to different coffee constituents may provide a deeper understanding of this reassuring result and the real role of coffee on cancer risk.
\end{abstract}

Coffee; Gastric Neoplasms; Meta-analysis
Francisco Botelho 1

Nuno Lunet 1

Henrique Barros ${ }^{1}$

\section{Introduction}

Coffee is one of the most popular beverages, with a yearly world average consumption of $1.1 \mathrm{~kg}$ per capita, which reaches $4.5 \mathrm{~kg}$ in industrialized countries 1 .

Roasted coffee is a complex mixture of more than a thousand chemicals. These constituents have been described as having genotoxic and mutagenic properties, but also antimutagenic and antioxidant activities and the capacity to inhibit cancer-promoting agents 2,3. Caffeine appears to disturb cell cycle checkpoint integrity, alter mechanisms of DNA repair, modify the apoptotic response, and potentiate a variety of DNA-damaging agents 2,3 . However, it can also inhibit carcinogenesis in vivo and alter carcinogen metabolism, decreasing the cytotoxic, cytostatic, or mutagenic activity of aromatic DNA-damaging compounds through a decrease in the concentration of free aromatic procarcinogens available for cytochrome activation 4,5. The protective effects of coffee were partially ascribed to the potential for kahweol and cafestol palmitates to convert rapid acetylators into a slow acetylator phenotype 6 . These diterpenes may also have anti-inflammatory and anti-carcinogenic properties by interfering with nitric oxide 7 , prostaglandin E2 production, and cyclooxigenase- 2 expression 8 . The antioxidant attributes of coffee may contribute to a protective role against cancer 9,10 . 
These contrasting effects parallel the results of previous epidemiological studies that disclosed no clear-cut effect of coffee consumption on cancer. Reviews and meta-analyses of available studies showed a $20 \%$ increased urinary tract cancer risk in coffee consumers 11 , no effect on pancreatic cancer risk 12 , and a $25 \%$ reduction in colorectal cancer risk 13,14.

Investigation of other cancer sites has been less extensive. An earlier review of the association between coffee and gastric cancer identified two cohort studies and nine case-control studies published until 199615 and underscored the contradictory nature of the results. Coffee is such a common exposure that any small effect can result in a large population impact. Therefore, we systematically reviewed and performed a meta-analysis of the published data addressing the association between coffee consumption and gastric cancer.

\section{Material and methods}

We performed a systematic review of case-control and cohort studies evaluating the association between coffee consumption and gastric cancer. We used PubMed (http://www.ncbi.nlm. nih.gov/entrez) to identify studies published through December 2004, under the searching expression “(stomach cancer OR gastric cancer OR cardia cancer) AND (coffee OR lifestyle OR tea)". The reference lists provided by the identified papers was additionally hand-searched. We evaluated full papers published in English, Spanish, French, Portuguese, and Italian, and English abstracts of full papers written in other languages.

Two reviewers extracted information from each study following a previously defined data collection procedure. Discrepancies in the evaluation of the articles were resolved by consensus, involving a third researcher. The protocol for data extraction covered: study design (case-control, population-based or hospital-based, or cohort, with respective follow-up time; number of subjects); histological confirmation of cases; histological type and location of gastric neoplasias; evaluation of exposure (interviewer or self-administered questionnaire as measuring instrument; type of coffee, reference class, exposure strata, timing of exposure); risk estimates for the association between coffee consumption and gastric cancer, considering two exposure levels (lowest vs. highest); precision estimates (confidence intervals, $P$ values, and number of participants in each exposure category); control of confounding factors; and country of origin.
In one study 16 there were only one case and one control in the upper exposure category and we opted for the results based on the immediate class of consumption.

We excluded from meta-analysis studies that did not provide relative risk estimates and respective variance, or the information needed to calculate it. When a study provided more than one estimate, we selected the one adjusted for the largest set of variables, and when results were available according to gender or ethnicity we included all estimates in the final analysis as if obtained from different studies.

Combined risk estimates and 95\% confidence intervals were computed using the random effects method, and statistical tests for homogeneity 17 were performed. Heterogeneity was investigated by subgroup analysis, looking at the magnitude of the combined risk estimates in each stratum as well as to the respective tests of heterogeneity, and meta-regression, to assess the independent contribution of each variable to explain heterogeneity. Publication bias was examined through funnel plot visual analysis, the Begg adjusted rank correlation test 18 , and the Egger's regression asymmetry test 19. A 0.1 level of significance was used in the statistical tests. The software STATA version 8.0 (Stata Corporation, College Station, USA) was used in all analyses.

\section{Results}

We identified 40 publications. The English abstracts of two articles published in Chinese 20,21, two in Russian 22,23, one in Japanese 24 and one in Serbian 25 provided insufficient information to be included in the final analysis. One of these studies 25 described a negative correlation and another stated that in large amounts coffee increased the risk 23 . Three publications $26,27,28$ provided no quantitative risk estimates but stated that no statistically significant association was found. One study 29 only stated the finding of a non-statistically significant association in males, and a significant association in females (hazard ratio $=2.54$ ) without providing any further statistical estimates. Two studies 30,31 were later reported in more detail 32,33 , and the more recent publications were used. Four reports were excluded from analysis because tea and coffee were considered combined $(\mathrm{OR}=$ 3.2) 34 , coffee assessed as a preference $(\mathrm{OR}=1.02)$ 35 , only cardia cancers $(\mathrm{OR}=1.3) 36$ were evaluated, or results were presented separately for intestinal $(\mathrm{OR}=0.50)$ and diffuse $(\mathrm{OR}=1.98)$ type gastric cancer using the same control group 37 . 
One case-control study was not considered for meta-analysis because no precision estimates were available 38 . Table 1 briefly characterizes the 17 studies excluded from the final analysis.

Twenty-three studies 16,32,33,39,40,41,42,43,44,45, $46,47,48,49,50,51,52,53,54,55,56,57,58$ remained for quantitative data synthesis (Figure 1 and Table 2). There were 7 cohort studies, with follow-ups ranging from 4.3 to 15 years, and 16 case-control studies, 10 hospital-based, 5 populationbased, and one with no specified study base. Seven studies were European, 5 were conducted in the USA ( 3 on Japanese in Hawaii), and 5 in Japan. The remaining studies described other Asian $(n=4)$ and South American samples $(n=2)$.

Fourteen studies used only histologically confirmed cases, two 40,50 stated that respectively "most" and $90.6 \%$ of cases had histological confirmation, five 46,52,54,56,57 only indicated having used cancer registries to identify cases, and two 16,58 did not provide this information.

Six studies $45,47,52,54,55,57$ used a self-administered questionnaire, one 16 did not specify the measuring instrument, and the remaining 16 collected information by interview. Among the 16 case-control studies, three evaluated coffee exposure five or more years prior to the interview $44,48,51$, two did not specify the timing of exposure 16,42, and the rest assessed coffee consumption within five years before onset of disease. In 14 studies, the reference category was non-coffee drinkers, but 9 included subjects drinking more than one cup of coffee per week in the reference group. Nine studies presented results considering only two groups of exposure, and 14 defined three or more categories of consumption. Only two reports specified the type of coffee consumed as being Turkish coffee 16 and caffeine-containing coffee 30 . One study evaluated the consumption of hot coffee 32 .

From 9 studies only crude risk estimates could be used, 10 controlled for the potential confounding effect of tobacco smoking, and 4 provided results adjusted for the consumption of fruit or vegetables.

When all studies were combined, coffee intake showed no effect on gastric cancer $(\mathrm{OR}=$ 0.97; 95\%CI: 0.86-1.09, heterogeneity test: $\mathrm{p}=$ 0.08) (Figure 1).

The combined risk estimate was 1.02 (95\%CI: $0.76-1.37$, heterogeneity test: $\mathrm{p}=0.12$ ) for cohort studies, 0.90 for population-based casecontrol studies (95\%CI: 0.70-1.15, heterogeneity test: $p=0.19$ ), and 0.97 for hospital-based case-control studies (95\%CI: 0.83-1.13, heterogeneity test: $\mathrm{p}=0.14$ ).

The studies that adjusted coffee effect for smoking resulted in a combined odds ratio of
0.91 (95\%CI: 0.75-1.11, heterogeneity test: $\mathrm{p}=$ 0.02 ), and 0.94 (95\%CI: 0.79-1.12, heterogeneity test: $\mathrm{p}=0.99$ ) for the studies adjusting for fruit or vegetable consumption. The combined risk estimates including only the studies with all cases histologically confirmed was 0.97 (95\%CI: 0.84-1.12, heterogeneity test: $p=0.06$ ).

The common odds ratio for the 9 studies including coffee drinkers in the reference category was 0.86 (95\%CI: 0.67-1.11, heterogeneity test: $\mathrm{p}=0.007)$ and 1.02 (95\%CI: 0.90-1.14, heterogeneity test: $\mathrm{p}=0.77$ ) for the remaining 14 studies. The association was 1.11 (95\%CI: 0.951.30 , heterogeneity test: $\mathrm{p}=0.37$ ) when considering only the studies that described exposure to coffee in two categories and 0.89 (95\%CI: 0.76-1.03, heterogeneity test: $\mathrm{p}=0.15$ ) for those using more strata. The OR was $1.00(95 \% \mathrm{CI}$ : $0.81-1.23$, heterogeneity test: $\mathrm{p}=0.25$ ) for the studies using a self-administered questionnaire, and 0.96 (95\%CI: 0.83-1.12, heterogeneity test: $p=0.06$ ) for those assessing exposure by interview. Among the case-control studies, the combined risk estimate was 0.93 (95\%CI: 0.66-1.31, heterogeneity test: $\mathrm{p}=0.05$ ) for those assessing exposure five or more years before interview, and 0.95 (95\%CI: 0.83-1.09, heterogeneity test: $p=0.31$ ) when coffee consumption was evaluated closer to the time of the study.

The OR was 1.26 (95\%CI: 1.02-1.57, heterogeneity test: $\mathrm{p}=0.59)$ when considering studies conducted in the USA (OR $=1.28$, 95\%CI: 0.99 1.67 , for those three conducted in populations of Japanese ancestry). It was 0.97 (95\%CI: 0.821.14 , heterogeneity test: $\mathrm{p}=0.52$ ) for the 5 Japanese studies, 0.98 (95\%CI: 0.81-1.17, heterogeneity test $\mathrm{p}=0.34$ ) for the 7 studies from Europe, and 0.64 (95\%CI: 0.47-0.86, heterogeneity test: $\mathrm{p}=0.27$ ) for the two studies from South America.

A multivariate analysis including all the above variables showed that risk estimates differed significantly according to country of origin, with North America studies presenting a significantly higher risk (coefficient $=0.45, \mathrm{p}=$ 0.003), number of exposure strata (coefficient $=$ $-0.21, p=0.06$, for 3 or more groups compared with two), and reference category (coefficient = $-0.27, p=0.02$, for some drinking in reference group compared to non-exposed to coffee).

Neither visual inspection nor tests of statistical significance resulted in funnel plot asymmetry (Figure 2).

As described above, most of the studies excluded from the meta-analysis were not considered simply because they did not provide the necessary risk and precision estimates, were duplicate publications, or analyzed exposures 
Table 1

Summary characteristics of studies excluded from the meta-analysis.

\begin{tabular}{|c|c|c|c|c|c|c|c|}
\hline Reference & $\begin{array}{l}\text { Publication } \\
\text { year }\end{array}$ & Country & Type of study & $\begin{array}{l}\text { Coffee ("highest" } \\
\text { vs. "lowest") }\end{array}$ & OR $(95 \% \mathrm{Cl})$ & $\begin{array}{l}\text { Control for } \\
\text { confounders }\end{array}$ & $\begin{array}{l}\text { Reason for } \\
\text { exclusion }\end{array}$ \\
\hline Graham et al. 26 & 1967 & USA & $\begin{array}{l}\text { Case-control } \\
\text { Hospital based }\end{array}$ & ns & $\begin{array}{l}\text { No statistically } \\
\text { significant association }\end{array}$ & ns & $\begin{array}{l}\text { Does not } \\
\text { present any results }\end{array}$ \\
\hline Graham et al. 27 & 1972 & USA & $\begin{array}{l}\text { Case-control } \\
\text { Hospital based }\end{array}$ & ns & $\begin{array}{l}\text { No statistically } \\
\text { significant association }\end{array}$ & ns & $\begin{array}{l}\text { Does not present } \\
\text { any results }\end{array}$ \\
\hline Salimov 22 & 1984 & USSR & - & - & - & - & Article in Russian \\
\hline $\begin{array}{l}\text { Trichopoulos } \\
\text { et al. } 34\end{array}$ & 1985 & Greece & $\begin{array}{l}\text { Case-control } \\
\text { Hospital based }\end{array}$ & Quintiles & $3.2(0.39-37.82)^{\star}$ & ns & $\begin{array}{l}\text { Combines coffee } \\
\text { and tea }\end{array}$ \\
\hline Risch et al. 28 & 1985 & Canada & $\begin{array}{l}\text { Case-control } \\
\text { Population based }\end{array}$ & ns & "Little relationship" & ns & $\begin{array}{l}\text { Does not present } \\
\text { any results }\end{array}$ \\
\hline Tajima et al. 38 & 1985 & Japan & $\begin{array}{l}\text { Case-control } \\
\text { Hospital based }\end{array}$ & $\begin{array}{l}\text { Every day vs. } \\
\text { no drinking habit }\end{array}$ & 1.02 & Age, sex & $\begin{array}{l}\text { Does not present } \\
\text { precision estimates }\end{array}$ \\
\hline $\begin{array}{l}\text { Tajima \& } \\
\text { Tominaga } 24\end{array}$ & 1986 & Japan & $\begin{array}{l}\text { Case-control } \\
\text { Hospital based }\end{array}$ & - & - & - & Article in Japanese \\
\hline $\begin{array}{l}\text { La Vecchia } \\
\text { et al. } 30\end{array}$ & 1987 & Italy & $\begin{array}{l}\text { Case-control } \\
\text { Hospital based }\end{array}$ & Tertiles & 1.00 & Age, sex & $\begin{array}{l}\text { Same study as } \\
\text { La Vecchia et al. } 32\end{array}$ \\
\hline $\begin{array}{l}\text { Jarebinski } \\
\text { et al. } 23\end{array}$ & 1989 & Yugoslavia & $\begin{array}{l}\text { Case-control } \\
\text { Hospital based }\end{array}$ & - & $\begin{array}{l}\text { "coffee in large } \\
\text { amounts... } \\
\text {...increases the risk" }\end{array}$ & - & Article in Russian \\
\hline $\begin{array}{l}\text { Jarebinski } \\
\text { et al. } 25\end{array}$ & 1992 & Yugoslavia & Case-control & - & "negative correlation" & - & Article in Serbian \\
\hline $\begin{array}{l}\text { Hoshiyama \& } \\
\text { Sasaba } 31\end{array}$ & 1992 & Japan & $\begin{array}{l}\text { Case-control } \\
\text { Population based }\end{array}$ & $\begin{array}{l}\geq 10 \text { cups/week vs. } \\
\leq 1 \mathrm{cup} / \text { week }\end{array}$ & $0.8(0.5-1.3)$ & $\begin{array}{l}\text { Age, sex, } \\
\text { smoking, } \\
\text { administrative } \\
\text { division }\end{array}$ & $\begin{array}{l}\text { Same as Hoshiyama } \\
\& \text { Sasaba } 33\end{array}$ \\
\hline Ye et al. 20 & 1998 & China & Case-control & - & - & - & Article in Chinese \\
\hline Komoto et al. 37 & 1998 & Japan & $\begin{array}{l}\text { Case-control } \\
\text { Hospital based }\end{array}$ & $\begin{array}{l}\text { Consumption vs. } \\
\text { non-consumption }\end{array}$ & $\begin{array}{l}0.50(0.24-1.01) \\
\text { Intestinal } \\
1.98(0.71-5.46) \\
\text { Diffuse }\end{array}$ & $\begin{array}{l}\text { H. pylori } \\
\text { infection, } \\
\text { alcohol, } \\
\text { smoking, } \\
\text { blood group, } \\
\text { family history } \\
\text { of gastric } \\
\text { carcinoma }\end{array}$ & $\begin{array}{l}\text { Stratified in intestinal } \\
\text { and diffuse without } \\
\text { any global analysis }\end{array}$ \\
\hline Wang et al. 21 & 1999 & China & Case-control & - & - & - & Article in Chinese \\
\hline Terry et al. 36 & 2000 & Sweden & $\begin{array}{l}\text { Case-control } \\
\text { Population based }\end{array}$ & Quartiles & $1.3(0.8-2.0)$ & $\begin{array}{l}\text { Age, sex, } \\
\text { body mass } \\
\text { index, total } \\
\text { energy, energy } \\
\text { adjusted alcohol, } \\
\text { fruits and } \\
\text { vegetable } \\
\text { intake, } \\
\text { smoking, use } \\
\text { of anti-acids }\end{array}$ & 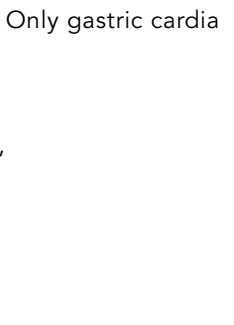 \\
\hline Huang et al. 35 & 2000 & Japan & $\begin{array}{l}\text { Controls were } \\
\text { hospital visitors }\end{array}$ & $\begin{array}{l}\text { Preference } \\
\text { for coffee }\end{array}$ & $1.02(0.83-1.24)$ & Age, sex & $\begin{array}{l}\text { Exposure was } \\
\text { preference for coffee }\end{array}$ \\
\hline Nagata et al. 29 & 2002 & Japan & $\begin{array}{l}\text { Cohort (mortality) } \\
\text { Follow-up: } 7 \text { years }\end{array}$ & $\begin{array}{l}\text { Daily vs. rare/ } \\
\text { never }\end{array}$ & $\begin{array}{l}\text { No statistically } \\
\text { significant } \\
\text { association (men) } \\
\text { Statistically significant } \\
\text { association, HR=2.54 } \\
\text { (women) }\end{array}$ & $\begin{array}{l}\text { Age, total } \\
\text { energy }\end{array}$ & $\begin{array}{l}\text { Does not present } \\
\text { precision estimates } \\
\text { Caffeinated coffee }\end{array}$ \\
\hline
\end{tabular}

ns $=$ not specified

* estimated using data available in the article. 
Meta-analysis of studies evaluating the association between coffee consumption and gastric cancer*.

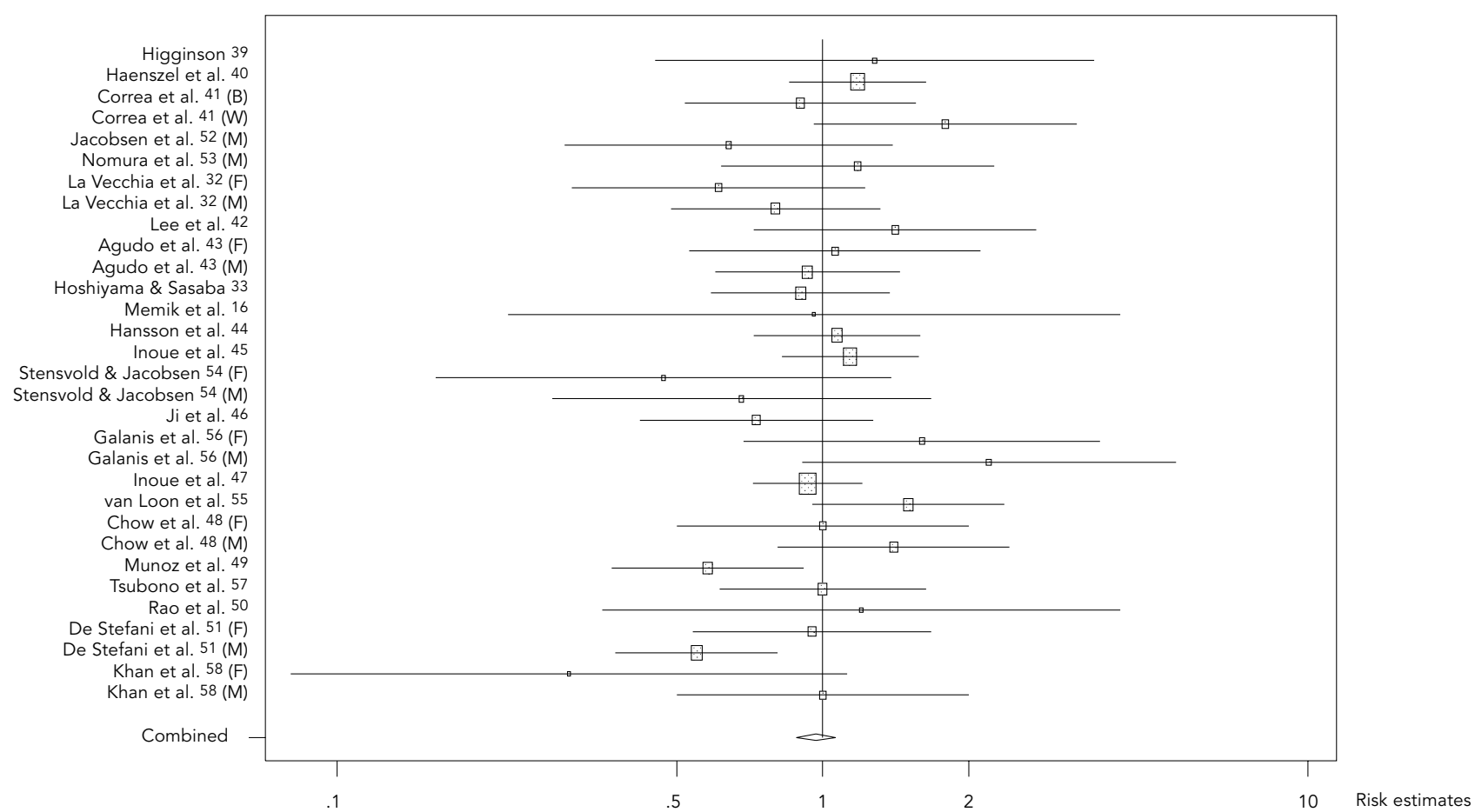

* Heterogeneity test $(p=0.08)$

$\mathrm{M}=$ Male; $\mathrm{F}=$ Female; $\mathrm{B}=$ Black; $\mathrm{W}=$ White.

to coffee and tea together. Relaxing the inclusion criteria to accommodate the remaining studies $35,36,37$ the summary risk estimate was 1.05 (95\%CI: $0.90-1.23$, heterogeneity test: $\mathrm{p}<$ 0.001 ), similar to that obtained in our main analysis.

\section{Discussion}

This meta-analysis of studies published during the last three decades showed no overall effect of coffee consumption on gastric cancer risk. However, we observed substantial methodological differences between studies that have potential effect on the risk estimates.

Most studies presented results on the association between coffee and gastric cancer as secondary data analysis or part of confounder evaluation $17,33,38,39,40,41,42,46,47,49,50,51,56,57,58$, and it is unlikely that this specific result influenced publication. The studies excluded from the analysis presented non-significant associa- tions between coffee and gastric cancer, and after changing our inclusion criteria, enlarging the number of studies combined, the results remained virtually unchanged. Publication bias is an improbable cause of our findings, as supported by the funnel plot analysis and the result of the regression asymmetry test, and it is unlikely that unpublished results would change our conclusions, because the bias is due to the over-publication of positive findings 59 .

In this analysis, several sources of heterogeneity are likely, even if most risk estimates from individual reports were not significantly different as assessed by statistical tests.

In case-control studies, coffee consumption among controls may not represent the target population, and bias is even more probable with hospital controls. However, considering the results from hospital- and population-based studies, this appears unlikely. Symptoms, namely heartburn, are associated with coffee intake, and cases might avoid coffee. This differential information bias could lead to a misleading 
Table 2

Summary characteristics of studies included in the meta-analysis.

\begin{tabular}{|c|c|c|c|c|c|c|c|c|c|}
\hline Reference & $\begin{array}{l}\text { Publi- } \\
\text { cation } \\
\text { year }\end{array}$ & Country & $\begin{array}{l}\text { Study } \\
\text { charac- } \\
\text { teristics }\end{array}$ & $\begin{array}{l}\text { Coffee } \\
\text { ("highest" } \\
\text { vs. "lowest") }\end{array}$ & $\begin{array}{l}\text { Number } \\
\text { of subjects }\end{array}$ & $\begin{array}{l}\text { Evaluation } \\
\text { of coffee } \\
\text { consumption }\end{array}$ & $\begin{array}{l}\text { OR/RR } \\
(95 \% \mathrm{Cl})\end{array}$ & $\begin{array}{l}\text { Control for } \\
\text { confounders }\end{array}$ & Notes \\
\hline Higginson 39 & 1966 & USA & $\begin{array}{l}\text { Case-control } \\
\text { Hospital based }\end{array}$ & $\begin{array}{l}\text { > } 3 \text { cups/day vs. } \\
\text { never/irregularly }\end{array}$ & $\begin{array}{l}54: 152^{\star}, \star \star \\
5: 18^{\star}, \star \star \star\end{array}$ & $\begin{array}{l}\text { Previous } 2 \\
\text { years }\end{array}$ & $\begin{array}{l}1.28 \\
(0.45-3.61)^{\#}\end{array}$ & No\# & \\
\hline $\begin{array}{l}\text { Haenszel } \\
\text { et al. } 40\end{array}$ & 1972 & USA & $\begin{array}{l}\text { Case-control } \\
\text { Hospital based }\end{array}$ & $\begin{array}{l}\geq 2 \text { cups/day vs. } \\
<2 \text { cups/day }\end{array}$ & $\begin{array}{l}102: 186^{*, \star *} \\
118: 253^{*, \star \star \star}\end{array}$ & Current & $\begin{array}{l}1.18 \\
(0.85-1.63)^{\#} \\
2.0 \text { (Issei) } \\
0.74 \text { (Nisei) }\end{array}$ & $\begin{array}{l}\text { Matching for sex } \\
\text { Sex- and nativity- } \\
\text { adjusted OR } \\
\text { is } 1.21, \text { but } \\
\text { no precision } \\
\text { estimate is } \\
\text { available }\end{array}$ & $\begin{array}{l}\text { Japanese in Hawaii } \\
\text { No differences by } \\
\text { histological type }\end{array}$ \\
\hline $\begin{array}{l}\text { Correa } \\
\text { et al. } 41\end{array}$ & 1985 & USA & $\begin{array}{l}\text { Case-control } \\
\text { Hospital based }\end{array}$ & $\begin{array}{l}\text { Above median } \\
\text { vs. below } \\
\text { median }\end{array}$ & 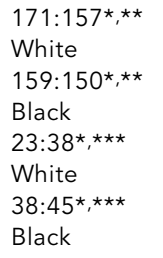 & $\begin{array}{l}\text { Before } \\
\text { illness }\end{array}$ & $\begin{array}{l}1.79 \\
(0.96-3.35) \\
\text { White } \\
0.9 \\
(0.52-1.56) \\
\text { Black }\end{array}$ & $\begin{array}{l}\text { Age, sex, } \\
\text { education, } \\
\text { income, tobacco, } \\
\text { and alcohol use }\end{array}$ & \\
\hline $\begin{array}{l}\text { La Vecchia } \\
\text { et al. } 32\end{array}$ & 1989 & Italy & $\begin{array}{l}\text { Case-control } \\
\text { Hospital based }\end{array}$ & $\begin{array}{l}\geq 4 \text { cups/day } \\
\text { vs. } 0 \text { cups/day }\end{array}$ & 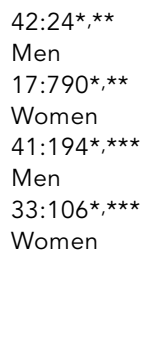 & Current & $\begin{array}{l}0.80 \\
(0.49-1.32) \\
\text { Men\# } \\
0.61 \\
(0.30-1.21) \\
\text { Women\# }\end{array}$ & $\begin{array}{l}\text { No } \\
\text { OR adjusted for } \\
\text { age, sex, social } \\
\text { class, education, } \\
\text { marital status, } \\
\text { smoking, alcohol } \\
\text { consumption } \\
\text { ( } \geq 3 \text { vs. } \leq 1 \text { cups/ } \\
\text { day) is } 1.26 \text {, but } \\
\text { no precision } \\
\text { estimate is availabl }\end{array}$ & $\begin{array}{l}\text { Caffeine-containing } \\
\text { coffee } \\
\text { e }\end{array}$ \\
\hline Lee et al. 42 & 1990 & Taiwan & $\begin{array}{l}\text { Case-control } \\
\text { Hospital based }\end{array}$ & $\begin{array}{l}\text { Drinker vs. } \\
\text { non-drinker }\end{array}$ & $\begin{array}{l}14: 39 *, \star \star \\
196: 771^{\star, \star \star \star}\end{array}$ & ns & $\begin{array}{l}1.41 \\
(0.72-2.75)^{\#}\end{array}$ & $\begin{array}{l}\text { Matching for age } \\
\text { and sex }\end{array}$ & \\
\hline $\begin{array}{l}\text { Hoshiyama } \\
\text { \& Sasaba } 33\end{array}$ & 1992 & Japan & $\begin{array}{l}\text { Case-control } \\
\text { Population } \\
\text { based }\end{array}$ & $\begin{array}{l}\geq 10 \mathrm{cups} / \text { week } \\
\text { vs. } \leq 1 \mathrm{cup} / \text { week }\end{array}$ & 251:483*,\#\# & Current & $0.9(0.6-1.4)$ & $\begin{array}{l}\text { Age, smoking, } \\
\text { dietary items } \\
\text { (fruit, vegetables, } \\
\text { preference for } \\
\text { salty foods etc.) }\end{array}$ & Hot coffee \\
\hline $\begin{array}{l}\text { Memik } \\
\text { et al. } 16\end{array}$ & 1992 & Turkey & Case-control & $\begin{array}{l}2-3 \text { cups/day vs. } \\
\leq 1 \text { cup/day }\end{array}$ & $\begin{array}{l}3: 24^{\star}, \star \star \\
76: 584^{\star}, \star \star \star\end{array}$ & ns & $\begin{array}{l}0.96 \\
(0.18-3.28) \#\end{array}$ & $\begin{array}{l}\text { Matching } \\
\text { for age }\end{array}$ & $\begin{array}{l}\text { Turkish coffee } \\
\text { No methods section }\end{array}$ \\
\hline $\begin{array}{l}\text { Agudo } \\
\text { et al. } 43\end{array}$ & 1992 & Spain & $\begin{array}{l}\text { Case-control } \\
\text { Hospital based }\end{array}$ & $\begin{array}{l}\text { Drinker vs. } \\
\text { non-drinker }\end{array}$ & $228: 227^{\star}$,\#\# & $\begin{array}{l}1 \text { year } \\
\text { before }\end{array}$ & $\begin{array}{l}0.93 \\
(0.60-1.44) \\
\text { Men } \\
1.06 \\
(0.53-2.11) \\
\text { Women }\end{array}$ & $\begin{array}{l}\text { Age, sex, area } \\
\text { residence, total } \\
\text { calories, fruits, } \\
\text { vegetables, } \\
\text { cold cuts, } \\
\text { preserved fish }\end{array}$ & \\
\hline $\begin{array}{l}\text { Hansson } \\
\text { et al. } 44\end{array}$ & 1993 & Sweden & $\begin{array}{l}\text { Case-control } \\
\text { Population } \\
\text { based }\end{array}$ & $\begin{array}{l}\geq 3,100 \mathrm{ml} / \text { week } \\
\text { vs. none }\end{array}$ & $338: 669 *$ *\# & $\begin{array}{l}20 \text { years } \\
\text { before }\end{array}$ & $\begin{array}{l}1.07 \\
(0.72-1.59)\end{array}$ & $\begin{array}{l}\text { Age, sex, } \\
\text { social class }\end{array}$ & $\begin{array}{l}\text { Exposure during } \\
\text { adolescence } \\
\mathrm{OR}=1.35(95 \% \mathrm{Cl} \text { : } \\
0.84-2.16)\end{array}$ \\
\hline
\end{tabular}

(continues) 
Table 2 (continued)

\begin{tabular}{|c|c|c|c|c|c|c|c|c|c|}
\hline Reference & $\begin{array}{l}\text { Publi- } \\
\text { cation } \\
\text { year }\end{array}$ & Country & $\begin{array}{l}\text { Study } \\
\text { charac- } \\
\text { teristics }\end{array}$ & $\begin{array}{l}\text { Coffee } \\
\text { ("highest" } \\
\text { vs. "lowest") }\end{array}$ & $\begin{array}{l}\text { Number } \\
\text { of subjects }\end{array}$ & $\begin{array}{l}\text { Evaluation } \\
\text { of coffee } \\
\text { consumption }\end{array}$ & $\begin{array}{l}\text { OR/RR } \\
(95 \% \mathrm{Cl})\end{array}$ & $\begin{array}{l}\text { Control for } \\
\text { confounders }\end{array}$ & Notes \\
\hline $\begin{array}{l}\text { Inoue } \\
\text { et al. } 45\end{array}$ & 1994 & Japan & $\begin{array}{l}\text { Case-control } \\
\text { Hospital based }\end{array}$ & $\begin{array}{l}\text { Ever drinker vs. } \\
\text { non-drinker }\end{array}$ & 668/668*,\#\# & $\begin{array}{l}\text { Before } \\
\text { illness }\end{array}$ & $\begin{array}{l}1.14 \\
(0.82-1.57)\end{array}$ & Sex & $\begin{array}{l}\text { Cardia, OR =0.94, } \\
95 \% \mathrm{Cl}: 0.52-1.69) \\
\text { Middle stomach, } \\
\text { OR }=1.04,95 \% \mathrm{Cl} \text { : } \\
0.66-1.64) ; \text { Antrum, } \\
\text { OR }=1.32,95 \% \mathrm{Cl} \\
0.88-1.97)\end{array}$ \\
\hline Ji et al. 46 & 1996 & China & $\begin{array}{l}\text { Case-control } \\
\text { Population } \\
\text { based }\end{array}$ & $\begin{array}{l}\text { Drinker vs. } \\
\text { non-drinker }\end{array}$ & $\begin{array}{l}21: 32^{\star, \star \star} \\
1103: \\
1217^{\star, \star \star \star}\end{array}$ & $\begin{array}{l}1 \text { year } \\
\text { before }\end{array}$ & $\begin{array}{l}0.73 \\
(0.42-1.27) \#\end{array}$ & $\begin{array}{l}\text { Matching for } \\
\text { age and sex }\end{array}$ & \\
\hline $\begin{array}{l}\text { Inoue } \\
\text { et al. } 47\end{array}$ & 1998 & Japan & $\begin{array}{l}\text { Case-control } \\
\text { Hospital based }\end{array}$ & $\begin{array}{l}\geq 3 \text { cups/day } \\
\text { vs. rarely }\end{array}$ & $\begin{array}{l}84: 1085^{\star, \star \star} \\
149: \\
1274^{\star, \star \star \star}\end{array}$ & $\begin{array}{l}\text { Before } \\
\text { illness }\end{array}$ & $\begin{array}{l}0.93 \\
(0.72-1.21)\end{array}$ & $\begin{array}{l}\text { Age, sex, } \\
\text { smoking, alcohol, } \\
\text { tea, physical } \\
\text { exercise, fruit, } \\
\text { beef, rice, year } \\
\text { and season at } \\
\text { first hospital visit }\end{array}$ & \\
\hline $\begin{array}{l}\text { Chow } \\
\text { et al. } 48\end{array}$ & 1999 & Poland & $\begin{array}{l}\text { Case-control } \\
\text { Population } \\
\text { based }\end{array}$ & $\begin{array}{l}\geq 7 \text { cups/week } \\
\text { vs. none }\end{array}$ & $\begin{array}{l}63: 68^{\star}, \star \star \\
\text { Women } \\
95: 85^{\star, \star \star} \\
\text { Men } \\
27: 39 \star, \star \star \star \\
\text { Women } \\
52: 65^{\star, \star \star \star} \\
\text { Men }\end{array}$ & $\begin{array}{l}5 \text { years } \\
\text { before }\end{array}$ & $\begin{array}{l}1.0 \\
(0.5-2.0) \\
\text { Women } \\
1.4 \\
(0.8-2.4) \\
\text { Men }\end{array}$ & $\begin{array}{l}\text { Age, smoking, } \\
\text { education, years } \\
\text { lived on a farm, } \\
\text { family history } \\
\text { of cancer }\end{array}$ & $\begin{array}{l}\text { No differences by } \\
\text { tumor location or } \\
\text { Lauren's histological } \\
\text { type }\end{array}$ \\
\hline $\begin{array}{l}\text { Munoz } \\
\text { et al. } 49\end{array}$ & 2001 & Venezuela & $\begin{array}{l}\text { Case-control } \\
\text { Population } \\
\text { based }\end{array}$ & Quartiles & 292:485*,\#\# & Current & $\begin{array}{l}0.58 \\
(0.37-0.92)\end{array}$ & $\begin{array}{l}\text { Age, sex, } \\
\text { smoking, alcohol, } \\
\text { total energy intake, } \\
\text { social class }\end{array}$ & \\
\hline $\begin{array}{l}\text { Rao } \\
\text { et al. } 50\end{array}$ & 2002 & India & $\begin{array}{l}\text { Case-control } \\
\text { Hospital based }\end{array}$ & $\begin{array}{l}\text { Daily vs. } \\
\text { never/rarely }\end{array}$ & $\begin{array}{l}3: 35^{\star, \star \star} \\
116: \\
1542^{\star, \star \star \star}\end{array}$ & Current & $1.2(0.3-3.5)$ & Age, sex & \\
\hline $\begin{array}{l}\text { De Stefani } \\
\text { et al. } 51\end{array}$ & 2004 & Uruguay & $\begin{array}{l}\text { Case-control } \\
\text { Hospital based }\end{array}$ & $\begin{array}{l}\text { Highest vs. } \\
\text { lowest tertile }\end{array}$ & 240:960*,\#\# & $\begin{array}{l}5 \text { years } \\
\text { before }\end{array}$ & $\begin{array}{l}0.55 \\
(0.38-0.82) \\
\text { Men } \\
0.95 \\
(0.54-1.67) \\
\text { Women }\end{array}$ & $\begin{array}{l}\text { Age, residence, } \\
\text { urban/rural } \\
\text { status, education, } \\
\text { body mass index, } \\
\text { smoking, alcohol, } \\
\text { total energy intake }\end{array}$ & \\
\hline $\begin{array}{l}\text { Jacobsen } \\
\text { et al. } 52\end{array}$ & 1986 & Norway & $\begin{array}{l}\text { Cohort } \\
\text { (incidence) } \\
\text { Follow-up: } \\
11.5 \text { yrs }\end{array}$ & $\begin{array}{l}\geq 7 \text { cups/day vs. } \\
\leq 2 \text { cups/day }\end{array}$ & $\begin{array}{l}\text { 10: } \\
3375 \# \# \#, \star \star \\
24: \\
1763 \# \# \#, \star \star \star\end{array}$ & Baseline & $\begin{array}{l}0.64 \\
p=0.13 \\
\text { Men }\end{array}$ & $\begin{array}{l}\text { Age, residence, } \\
\text { smoking. Results } \\
\text { age-, sex- and } \\
\text { residence-adjusted } \\
\text { are available } \\
(\mathrm{RR}=1.46, \\
\mathrm{P}=0.21)\end{array}$ & \\
\hline $\begin{array}{l}\text { Nomura } \\
\text { et al. } 53\end{array}$ & 1986 & USA & $\begin{array}{l}\text { Cohort } \\
\text { (incidence) } \\
\text { Follow-up: } \\
15 \text { yrs }\end{array}$ & $\begin{array}{l}\geq 5 \text { cups/day } \\
\text { vs. none }\end{array}$ & $\begin{array}{l}26 \#: \\
1,850 \# \# \#, \star \star \\
14 \#: \\
1,178 \# \# \#, \star \star \star\end{array}$ & Baseline & $\begin{array}{l}1.18 \\
(0.62-2.26)^{\#} \\
\text { Men }\end{array}$ & Age & $\begin{array}{l}\text { Japanese men } \\
\text { in Hawaii }\end{array}$ \\
\hline
\end{tabular}

(continues) 
Table 2 (continued)

\begin{tabular}{|c|c|c|c|c|c|c|c|c|c|}
\hline Reference & $\begin{array}{l}\text { Publi- } \\
\text { cation } \\
\text { year }\end{array}$ & Country & $\begin{array}{l}\text { Study } \\
\text { charac- } \\
\text { teristics }\end{array}$ & $\begin{array}{l}\text { Coffee } \\
\text { ("highest" } \\
\text { vs. "lowest") }\end{array}$ & $\begin{array}{l}\text { Number } \\
\text { of subjects }\end{array}$ & $\begin{array}{l}\text { Evaluation } \\
\text { of coffee } \\
\text { consumption }\end{array}$ & $\begin{array}{l}\text { OR/RR } \\
(95 \% \mathrm{Cl})\end{array}$ & $\begin{array}{l}\text { Control for } \\
\text { confounders }\end{array}$ & Notes \\
\hline $\begin{array}{l}\text { Stensvold \& } \\
\text { Jacobsen } 54\end{array}$ & 1994 & Norway & $\begin{array}{l}\text { Cohort } \\
\text { (incidence) } \\
\text { Follow-up: } \\
10.1 \text { yrs }\end{array}$ & $\begin{array}{l}\geq 7 \text { cups/day } \\
\text { vs. } \leq 2 \text { cups/day }\end{array}$ & $\begin{array}{l}14: \\
71,923 \S, \star \star \\
\text { Men } \\
6: 47,530 \S, \star \star \\
\text { Women } \\
7: 24,576 \S, \star \star \star \\
\text { Men } \\
7: 25,906 \S, \star \star \star \\
\text { Women }\end{array}$ & Baseline & $\begin{array}{l}0.68 \\
(0.28-1.69) \# \\
\text { Men } \\
0.47 \\
(0.16-1.39) \# \\
\text { Women }\end{array}$ & $\begin{array}{l}\text { No } \\
\text { RR adjusted for } \\
\text { age, smoking } \\
\text { and county of } \\
\text { residence is } 0.5 \\
\text { for men and } 0.5 \\
\text { for women, but } \\
\text { no precision } \\
\text { estimates are } \\
\text { available }\end{array}$ & \\
\hline $\begin{array}{l}\text { van Loon } \\
\text { et al. } 55\end{array}$ & 1998 & $\begin{array}{l}\text { Nether- } \\
\text { lands }\end{array}$ & $\begin{array}{l}\text { Cohort } \\
\text { (incidence) } \\
\text { Follow-up: } \\
4.3 \text { yrs }\end{array}$ & $\begin{array}{l}>4 \text { cups/day } \\
\text { vs. } \leq 3 \mathrm{cups} / \text { day }\end{array}$ & $\begin{array}{l}29: 216 \S \S, \star \star \\
117: \\
1,309 \S \S, \star \star \star\end{array}$ & Baseline & $\begin{array}{l}1.5 \\
(0.95-2.36)^{\#}\end{array}$ & No & \\
\hline $\begin{array}{l}\text { Galanis } \\
\text { et al. } 56\end{array}$ & 1998 & USA & $\begin{array}{l}\text { Cohort } \\
\text { (incidence) } \\
\text { Follow-up: } \\
14.8 \text { yrs }\end{array}$ & $\begin{array}{l}\geq 2 \text { cup/day } \\
\text { vs. none }\end{array}$ & $\begin{array}{l}32: \\
2,584 \# \# \#, \star \star \\
\text { Men } \\
19: 2703 \# \#, \star \star \\
\text { Women } \\
6: 1,647 \# \# \#, \star \star \star \\
\text { Men } \\
8: 1,868 \# \# \#, \star \star \star \\
\text { Women }\end{array}$ & $\begin{array}{l}\text { Baseline } \\
+ \\
+\end{array}$ & $\begin{array}{l}2.2 \\
(0.9-5.3) \\
\text { Men } \\
1.6 \\
(0.7-3.8) \\
\text { Women }\end{array}$ & $\begin{array}{l}\text { Age, sex, } \\
\text { education, } \\
\text { Japanese place } \\
\text { of birth, smoking } \\
\text { (only in men) }\end{array}$ & Japanese in Hawaii \\
\hline $\begin{array}{l}\text { Tsubono } \\
\text { et al. } 57\end{array}$ & 2001 & Japan & $\begin{array}{l}\text { Cohort } \\
\text { (incidence) } \\
\text { Follow-up: } 9 \text { yrs }\end{array}$ & $\begin{array}{l}\geq 3 \text { cups/day } \\
\text { vs. never }\end{array}$ & $\begin{array}{l}\text { 419: } \\
\text { 19,9748§,\#\# }\end{array}$ & Baseline & $1.0(0.6-1.6)$ & $\begin{array}{l}\text { Sex, age, tea, } \\
\text { smoking, } \\
\text { consumption of } \\
\text { alcohol, rice, } \\
\text { meat, vegetables, } \\
\text { fruits, bean-past } \\
\text { soup, type of } \\
\text { health insurance }\end{array}$ & \\
\hline $\begin{array}{l}\text { Khan } \\
\text { et al. } 58\end{array}$ & 2004 & Japan & $\begin{array}{l}\text { Cohort } \\
\text { (mortality) } \\
\text { Follow-up: Men, } \\
\text { 13.8 years; } \\
\text { Women, } \\
\text { 14.8 years }\end{array}$ & $\begin{array}{l}\geq \text { several times/ } \\
\text { week vs. } \leq \text { several } \\
\text { times/month }\end{array}$ & $\begin{array}{l}36 \text { cases\#\#| } \\
\text { in men } \\
927: 595 \S \S \S \\
\text { Men } \\
15 \text { cases\#\# } \\
\text { in women } \\
992: 641 \S \S \S \\
\text { Women }\end{array}$ & Baseline & $\begin{array}{l}1.0(0.5-2.0) \\
\text { Men } \\
0.3(0.1-1.4) \\
\text { Women }\end{array}$ & $\begin{array}{l}\text { Age, smoking } \\
\text { (men); age, } \\
\text { smoking, health } \\
\text { status, health } \\
\text { education, health } \\
\text { screening (women) }\end{array}$ & \\
\hline
\end{tabular}

ns = not specified.

* cases:controls;

** highest level of exposure;

$\star * \star$ lowest level of exposure;

\# estimated using data available in the article;

\#\# data stratified by exposure category not available;

\#\#\# cases:number of respondents;

$\S$ cases:person-years of follow-up;

$\S \S$ cases:subcohort subjects;

$\S \S \S$ subjects in reference:comparison groups. 
protective effect. Again, similar summary estimates for case-control and cohort studies argue against this hypothesis.

The classification of exposure differs considerably across the reviewed studies, and the results were shown to be significantly different according to the number of consumption categories evaluated or the characteristics of the reference class regarding consumption. The lower risk estimates when consumption is evaluated in several categories could be explained if coffee had a protective effect that would be stronger when the difference between the groups of highest and lowest exposure was larger. On the other hand, in opposition to what is observed, if coffee had some effect on cancer risk we would expect the combined estimates to approach the null when the reference class includes coffee drinkers, and this is likely to be a chance finding. The method of questionnaire administration and the timing of exposure evaluation did not significantly influence our results, and our conclusions of lack of positive association between coffee and cancer do not appear to be affected by the specificities of exposure evaluation.

In the meta-analysis, we opted for individual risk estimates based on exposure categories with different cut-off points, and a dose-response analysis would have given more information on the underlying association. However, more than one-third of the studies allowed no such analysis, and we opted to include the 9 studies providing data for only two consumption categories. This option proved to be adequate, since the combined risk estimates differed across studies presenting results for two or more groups of exposure.

Coffee consumption tends to be associated with tobacco smoking 54,60 , but many studies did not account for this potential confounding in the data analysis. Moreover, adding to residual confounding by tobacco smoking, other factors may be influencing the estimates for the association between coffee and gastric cancer, since non-coffee drinkers may differ from the general population of coffee drinkers concerning other exposures such as tea, alcohol, or fruit and vegetable intake 61,62 . The studies included in our review rarely considered confounding or interaction from these variables.

We observed differences in risk estimates according to the geographical origin of the study. Methodological options in study design did not explain such differences, but none of the reviewed studies considered in detail the characteristics of the coffee consumed. It is known that the type of coffee beans $63,64,65$,
Figure 2

Funnel plot of studies evaluating the association between gastric cancer and coffee consumption*.

Begg's funnel plot with pseudo $90 \%$ confidence limits

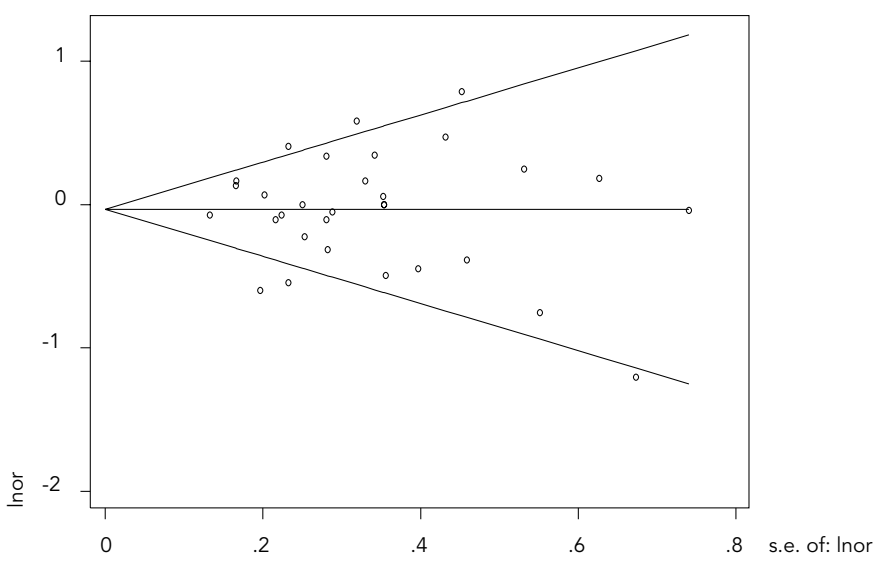

* Begg adjusted rank correlation test $(p=0.68)$, Egger's regression asymmetry test $(p=0.92)$.

roasting procedure 66,67 , and specific method of preparing the coffee can influence composition $68,69,70$. We can speculate on any potential adverse effect of specific constituents present in coffee consumed in North America. The underlying risk of gastric cancer in each population, international differences in the typical amount of coffee consumed, coffee type, or brewing method 70 may contribute to these differences. Studies presenting different risk estimates across strata of gender $48,51,58$, ethnicity 41 , or generation of Japanese migrants to Hawaii 40 favor this hypothesis of various exposures under the label coffee, but the alternate explanation of confounding seems sounder.

The complexity of coffee composition and the multiple social contexts underlying consumption make the evaluation of the effect of coffee on gastric cancer very difficult. Human experimental studies on such associations are unlikely, making observational studies the best available source of evidence on risk. Overall, it is reassuring that this meta-analysis showed no adverse effect of coffee associated with gastric cancer. Knowledge on the level of exposure to different coffee constituents may provide a deeper understanding of the real role of coffee on cancer risk and ultimately allow the design of safer beverages. 


\section{Resumo}

Efetuamos uma revisão sistemática dos estudos publicados avaliando a associação entre café e câncer de estômago. Identificamos estudos de coorte e caso-controle na PubMed e nas listas de referências. Foram obtidas estimativas conjuntas do risco por meta-análise de 23 estudos (método de efeitos aleatórios). A heterogeneidade foi explorada por estratificação e meta-regressão. $O$ odds ratio (OR) conjunto para a associação entre café e câncer gástrico (categoria de exposição mais elevada vs. mais baixa) foi de 0,97 (IC95\%: 0,86$1,09)$, semelhante para estudos de coorte $(O R=1,02$; IC95\%: 0,76-1,37) e caso-controle (populacional: $O R=$ 0,90; IC95\%: 0,70-1,15; hospitalar: OR =0,97; IC95\%: 0,83-1,13). O OR foi de 1,26 (IC95\%: 1,02-1,57) para cinco estudos efetuados nos Estados Unidos, 0,97 (IC95\%: 0,82-1,14) para cinco estudos japoneses, 0,98 (IC95\%: 0,81-1,17) para cinco estudos europeus, e 0,64 (IC95\%: 0,47-0,86) para dois estudos sul-americanos. Nesta meta-análise não observamos efeito significativo do consumo de café na ocorrência de câncer gástrico. Contudo, o conhecimento dos níveis de exposição a diferentes constituintes do café poderá permitir uma melhor compreensão deste resultado e o verdadeiro contributo do café para a ocorrência de câncer.

Café; Neoplasias Gástricas; Meta-análise

\section{Contributors}

F. Botelho was responsible for the searches in PubMed and the lists of references, extracted data from the articles, and wrote the first draft of the manuscript. N. Lunet extracted data from the articles, performed the statistical analysis of data, and contributed to drafting of the final manuscript. H. Barros extracted data from the articles and contributed to the drafting of the final manuscript. All authors approved the final version of the manuscript.

\section{Acknowledgements}

Nuno Lunet received a grant from Fundação para a Ciência e a Tecnologia (SFRH/BD/3293/2000).

\section{References}

1. Food and Agricultural Organization. Food balance sheets. http://www.fao.org/waicent/portal/statistics_en.asp (accessed on Apr/2004).

2. Nehlig A, Debry G. Potential genotoxic, mutagenic and antimutagenic effects of coffee: a review. $\mathrm{Mu}$ tat Res 1994; 317:145-62.

3. Porta M, Vioque J, Ayude D, Alguacil J, Jariod M, Ruiz L, et al. Coffee drinking: the rationale for treating it as a potential effect modifier of carcinogenic exposures. Eur J Epidemiol 2003; 18:289-98.

4. Kapuscinski J, Ardelt B, Piosik J, Zdunek M, Darzynkiewicz Z. The modulation of the DNAdamaging effect of polycyclic aromatic agents by xanthines. Part I. Reduction of cytostatic effects of quinacrine mustard by caffeine. Biochem Pharmacol 2002; 63:625-34.

5. Piosik J, Ulanowska K, Gwizdek-Wisniewska A, Czy A, Kapuscinski J, Wegrzyn G. Alleviation of mutagenic effects of polycyclic aromatic agents by caffeine and pentoxifylline. Mutat Res 2003; 530:47-57.

6. Huber WW, Teitel CH, Coles BF, King RS, Wiese FW, Kaderlik KR, et al. Potential chemoprotective effects of the coffee components kahweol and cafestol palmitates via modification of hepatic $\mathrm{N}$ acetyltransferase and glutathione S-transferase activities. Environ Mol Mutagen 2004; 44:265-76.

7. Kim JY, Jung KS, Lee KJ, Na HK, Chun HK, Kho $\mathrm{YH}$, et al. The coffee diterpene kahweol suppress the inducible nitric oxide synthase expression in macrophages. Cancer Lett 2004; 213:147-54.

8. Kim JY, Jung KS, Jeong HG. Suppressive effects of kahweol and cafestol on cyclooxygenase-2 expression in macrophages. FEBS Lett 2004; 569:321-6.

9. Natella F, Nardini M, Giannetti I, Dattilo C, Scaccini C. Coffee drinking influences plasma antioxidant capacity in humans. J Agric Food Chem 2002; 50:6211-6.

10. Svilaas A, Sakhi AK, Andersen LF, Svilaas T, Strom EC, Jacobs Jr. DR, et al. Intakes of antioxidants in coffee, wine, and vegetables are correlated with plasma carotenoids in humans. J Nutr 2004; 134: 562-7.

11. Zeegers M, Tan F, Goldbohm R, van den Brandt P. Are coffee and tea consumption associated with urinary tract risk? A systematic review and metaanalysis. Int J Epidemiol 2001; 30:353-62.

12. Tavani A, La Vecchia C. Coffee and cancer: a review of epidemiological studies, 1990-1999. Eur J Cancer Prev 2000; 9:241-56.

13. Giovannucci E. Meta-analysis of coffee consumption and risk of colorectal cancer. Am J Epidemiol 1998; 147:1043-52.

14. Tavani A, La Vecchia C. Coffee, decaffeinated coffee, tea and cancer of the colon and rectum: a review of epidemiological studies, 1990-2003. Cancer Causes Control 2004; 15:743-57.

15. World Cancer Research Fund/American Institute for Cancer Research. Food, nutrition and the prevention of cancer: a global perspective. Washington DC: World Cancer Research Fund/American Institute for Cancer Research; 1997.

16. Memik F, Nak SG, Gulten M, Ozturk M. Gastric carcinoma in northwestern Turkey: epidemiolog- 
ic characteristics. J Environ Pathol Toxicol Oncol 1992; 11:335-8.

17. Petiti DB. Meta-analysis, decision analysis, and cost effectiveness analysis: methods for quantitative synthesis in Medicine. 2nd Ed. Oxford: Oxford University Press; 2000.

18. Begg CB, Mazumdar M. Operating characteristics of a rank correlation test for publication bias. Biometrics 1994; 50:1088-101.

19. Egger M, Smith GD, Schieder M, Minder C. Bias in meta-analysis detected by a simple graphical test. BMJ 1997; 315:629-34.

20. Ye W, Yi Y, Luo R. A case-control study on diet and gastric cancer. Zhonghua Liu Xing Bing Xue Za Zhi 1998; 32:100-2.

21. Wang M, Guo C, Li M. A case-control study on the dietary risk factors of upper digestive tract cancer. Zhonghua Liu Xing Bing Xue Za Zhi 1999; 20:95-7.

22. Salimov TG. Effect of various social and life-style factors on the incidence of stomach disease. Klin Med (Mosk) 1984; 62:57-60.

23. Jarebinski M, Vlajinac H, Adanja B. An assessment of the interrelationship of biosocial factors and stomach cancer-the results of clinical anamnestic research in Belgrade. Ter Arkh 1989; 61:40-4.

24. Tajima K, Tominaga S. A comparative case-control study of stomach and large intestinal cancers. Gan No Rinsho 1986; 32:582-90.

25. Jarebinski M, Adanja B, Vlajinac H, Pekmezovic T, Sipetic S. Evaluation of the association of cancer of the esophagus, stomach and colon with habits of patients. Vojnosanit Pregl 1992; 49:19-24.

26. Graham S, Lilienfeld A, Tidings J. Dietary and purgation factors in the epidemiology of gastric cancer. Cancer 1967; 20:2224-34.

27. Graham S, Scholz W, Martino P. Alimentary factors in the epidemiology of gastric cancer. Cancer 1972; 30:927-38.

28. Risch HA, Jain M, Choi NW, Fodor G, Pfeiffer CJ, Howe GR, et al. Dietary factors and the incidence of cancer of the stomach. Am J Epidemiol 1985; 122:947-59.

29. Nagata C, Takatsuka N, Kawakami N, Shimizu H. A prospective cohort study of soy product intake and stomach cancer death. Br J Cancer 2002; 87: 31-6.

30. La Vecchia C, Negri E, Decarli A, D'Avanzo B, Franceschi S. A case-control study of diet and gastric cancer in northern Italy. Int J Cancer 1987; 40: 484-9.

31. Hoshiyama Y, Sasaba T. A case-control study of stomach cancer and its relation to diet, cigarettes, and alcohol consumption in Saitama Prefecture, Japan. Cancer Causes Control 1992; 3:441-8.

32. La Vecchia C, Ferraroni M, Negri E, D'Avanzo B, Decarli A, Levi F, et al. Coffee consumption and digestive tract cancers. Cancer Res 1989; 49:1049-51.

33. Hoshiyama Y, Sasaba T. A case-control study of single and multiple stomach cancers in Saitama prefecture, Japan. Jpn J Cancer Res 1992; 83:937-43.

34. Trichopoulos D, Ouranos G, Day NE, Tzonou A, Manousos O, Papadimitriou C, et al. Diet and cancer of the stomach: a case-control study in Greece. Int J Cancer 1985; 36:291-7.
35. Huang E, Tajima K, Hamajima N. Comparison of lifestyle and risk factors among Japanese with and without gastric cancer family history. Int J Cancer 2000; 86:421-4.

36. Terry P, Lagergren J, Wolk A, Nyren O. Reflux-inducing dietary factors and risk of adenocarcinoma of the esophagus and gastric cardia. Nutr Cancer 2000; 38:186-91.

37. Komoto K, Haruma K, Kamada T, Tanaka S, Yoshihara M, Sumii K, et al. Helicobacter pylori infection and gastric neoplasia: correlations with histological gastritis and tumor histology. Am J Gastroenterol 1998; 93:1271-6

38. Tajima K, Tominaga S. Dietary habits and gastrointestinal cancers: a comparative case-control study of stomach and large intestinal cancers in Nagoya, Japan. Jpn J Cancer Res 1985; 76:705-16.

39. Higginson J. Etiological factors in gastrointestinal cancer in man. J Natl Cancer Inst 1966; 37:527-4.

40. Haenszel W, Kurihara M, Segi M, Lee R. Stomach cancer among Japanese in Hawaii. J Natl Cancer Inst 1972; 49:969-88.

41. Correa P, Fontham E, Pickle L, Chen V, Lin Y, Haenszel W. Dietary determinants of gastric cancer in South Louisiana inhabitants. J Natl Cancer Inst 1985; 75:645-53.

42. Lee HH, Wu HY, Chuang YC, Chang AS, Chao HH, Chen KY, et al. Epidemiologic characteristics and multiple risk factors of stomach cancer in Taiwan. Anticancer Res 1990; 10:875-81.

43. Agudo A, González CA, Marcos G, Sanz M, Saigi E, Verge J, et al. Consumption of alcohol, coffee, and tobacco, and gastric cancer in Spain. Cancer Causes Control 1992; 3:137-43.

44. Hansson LE, Nyren O, Bergstrom R, Wolk A, Lindgren A, Baron J, et al. Diet and risk of gastric cancer. A population-based case-control study in Sweden. Int J Cancer 1993; 55:181-9.

45. Inoue M, Tajima K, Hirose K, Kuroishi T, Gao C, Kitoh T. Life-style and subsite of gastric cancer: joint effect of smoking and drinking habits. Int J Cancer 1994; 56:494-9.

46. Ji B, Chow W, Yang G, McLaughlin J, Gao R, Zheng $\mathrm{W}$, et al. The influence of cigarette smoking, alcohol, and green tea consumption on the risk of carcinoma of the cardia and distal stomach in Shanghai, China. Cancer 1996; 77:2449-56.

47. Inoue $\mathrm{M}$, Tajima $\mathrm{K}$, Hirose $\mathrm{K}$, Hamajima $\mathrm{N}$, Takezaki T, Kuroishi T, et al. Tea and coffee consumption and the risk of digestive tract cancers. Data from a comparative case-referent study in Japan. Cancer Causes Control 1998; 9:209-16.

48. Chow WH, Swanson CA, Lissowska J, Grovers FD, Sobin LH, Nasierowska-Guttmejer A, et al. Risk of stomach cancer in relation to consumption of cigarettes, alcohol, tea and coffee in Warsaw, Poland. Int J Cancer 1999; 81:871-6.

49. Munoz N, Plummer M, Vivas J, Moreno V, De Sanjose S, Lopez G, et al. A case-control study of gastric cancer in Venezuela. Int J Cancer 2001; 93: 417-23.

50. Rao D, Ganesh B, Dinshaw K, Mohandas K. A case-control study of stomach cancer in Mumbai, India. Int J Cancer 2002; 99:727-31. 
51. De Stefani E, Correa P, Boffetta P, Deneo-Pellegrini H, Ronco AL, Mendilaharsu M. Dietary patterns and risk of gastric cancer: a case-control study in Uruguay. Gastric Cancer 2004; 7:211-20.

52. Jacobsen B, Bjeike E, Kvale G, Heuch I. Coffee drinking, mortality, and cancer incidence: results from a Norwegian prospective study. J Natl Cancer Inst 1986; 76:823-31.

53. Nomura A, Heilbrun L, Stemmermann G. Prospective study of coffee consumption and the risk of cancer. J Natl Cancer Inst 1986; 76:587-90.

54. Stensvold I, Jacobsen BK. Coffee and cancer: a prospective study of 43,000 Norwegian men and women. Cancer Causes Control 1994; 5:401-8.

55. van Loon AJ, Goldbohm RA, van den Brandt PA. Socioeconomic status and stomach cancer incidence in men: results from The Netherlands Cohort Study. J Epidemiol Community Health 1998; 52:166-71.

56. Galanis DJ, Kolonel LN, Lee J, Nomura A. Intakes of selected foods and beverages and the incidence of gastric cancer among the Japanese residents of Hawaii: a prospective study. J Epidemiol 1998; 27:173-80.

57. Tsubono Y, Nishino Y, Komatsu S, Hsieh C, Kanemura S, Tsuji I, et al. Green tea and the risk of gastric cancer in Japan. N Engl J Med 2001; 344:632-6.

58. Khan MMH, Goto R, Kobayashi K, Suzumura S, Nagata Y, Sonoda T, et al. Dietary habits and cancer mortality among middle aged and older Japanese living in Hokkaido, Japan by cancer site and sex. Asian Pacific J Cancer Prev 2004; 5:58-65.

59. Egger M, Smith GD. Bias in location and selection of studies. BMJ 1998; 316:61-6.

60. Schreiber GB, Robins M, Maffeo CE, Masters MN, Bond AP, Morganstein D. Confounders contributing to the reported associations of coffee or caffeine with disease. Prev Med 1988; 17:295-309.
61. Klesges RC, Ray JW, Klesges LM. Caffeinated coffee and tea intake and its relationship to cigarette smoking: an analysis of the Second National Health and Nutrition Examination Survey (NHANES II). J Subst Abuse 1994; 6:407-18.

62. Schwarz B, Bischof HP, Kunze M. Coffee, tea, and lifestyle. Prev Med 1994; 23:377-84.

63. Richelle M, Tavazzi I, Offord E. Comparison of the antioxidant activity of commonly consumed polyphenolic beverages (coffee, cocoa, and tea) prepared per cup serving. J Agric Food Chem 2001; 49:3438-42.

64. Leoni LA, Soares LM, Oliveira PL. Ochratoxin A in Brazilian roasted and instant coffees. Food Addit Contam 2000; 17:867-70.

65. Lombaert GA, Pellaers P, Chettiar M, Lavalee D, Scott PM, Lau BP. Survey of Canadian retail coffees for ochratoxin A. Food Addit Contam 2002; 19:869-77.

66. Del Castillo MD, Ames JM, Gordon MH. Effect of roasting on the antioxidant activity of coffee brews. J Agric Food Chem 2002; 50:3698-703.

67. Romani S, Pinnavaia GG, Dalla Rosa M. Influence of roasting levels on ochratoxin A content in coffee. J Agric Food Chem 2003; 51:5168-71.

68. Ratnayake WM, Hollywood R, O'Grady E, Stavric B. Lipid content and composition of coffee brews prepared by different methods. Food Chem Toxicol 1993; 31:263-9.

69. Urgert R, Schulz AG, Katan MB. Effects of cafestol and kahweol from coffee grounds on serum lipids and serum liver enzymes in humans. Am J Clin Nutr 1995; 61:149-54.

70. Gross G, Jaccaud E, Huggett AC. Analysis of the content of the diterpenes cafestol and kahweol in coffee brews. Food Chem Toxicol 1997; 35:547-54.

71. Urgert R, de Groot CP. Consumption of unfiltered coffee brews in elderly Europeans. SENECA Investigators. Eur J Clin Nutr 1996; 50 Suppl 2:S101-4.

Submitted on 28/Mar/2005

Final version resubmitted on 31/Aug/2005

Approved on 27/Sep/2005 